\title{
Formación Continua de los Docentes de la Educación Infantil: ¿cuál debe ser el debate?
}

\author{
Ofelia Reveco Vergara' \\ 'Universidad Central de Chile (UCENTRAL), Santiago - Chile
}

RESUMEN - Formación Continua de los Docentes de la Educación Infantil: ¿̨cuál debe ser el debate? En la búsqueda por mejorar la calidad de los aprendizajes en los niños/as y jóvenes, en los últimos años los Estados han concretado múltiples iniciativas, por ejemplo: diseñar o renovar los currículos, dotar a las escuelas de mejores infraestructuras y de recursos didácticos, evaluar el desempeño de los/as docentes, mejorar o universalizar los denominados sistemas de medición de la calidad de la educación, ofrecer formación continua a los/as educadores y procesos de especialización. Respecto de la formación continua de los docentes, el artículo busca responder a la pregunta: ¿La falta o escasa especialización de las/os Educadoras Infantiles en un grupo etario o en un área específica del conocimiento es el problema o, este está constituido por un ámbito de la educación que ha quedado olvidado o se ha trabajado menos: lo formativo?

Palabras-clave: Formación Continua. Educación Infantil. Debates en Educación.

\begin{abstract}
Continuing Education of Early Childhood Education Teachers: what debate should it be? In the search for a better quality of youngsters and children's learning, over the last years different countries have been developing multiple initiatives, such as: to design or reformulate curriculums, to provide schools with better infrastructure and didactic resources, to evaluate the performance of teachers, to improve or to universalize the assessment systems of the qualities of education, to offer continuing education and specialization for teachers. In relation to the continuing education, this article tries to answer to the following question: Is the lack of or poor specialization in an age group or in a specific area of knowledge by early childhood education teachers the real problem, or is the problem related to an aspect of education that has been forgotten or less worked on: the education?
\end{abstract}

Keywords: Continuing Education. Early Childhood. Debates in Education.

Educação \& Realidade, Porto Alegre, v. 40, n. 4, p. 973-986, out./dez. 2015. 973 http://dx.doi.org/10.1590/2175-623651523 


\section{Introducción}

En la búsqueda por mejorar la calidad de los aprendizajes en los niños, niñas y jóvenes, en los últimos años los Estados han concretado múltiples iniciativas: diseñar o renovar los currículos, dotar a las escuelas de mejores infraestructuras y recursos didácticos, evaluar el desempeño de los/as docentes, influir en la formación inicial docente, mejorar o universalizar los denominados sistemas de medición de la calidad de la educación, generar las condiciones para la formación continua de los educadores y ofrecer procesos de especialización de las/os educadores.

En el caso de la Educación Infantil, en los últimos años, se ha puesto en la discusión y se ha avanzado en concretar la especialización de educadores en ciertos grupos etáreos o en determinadas áreas de contenidos. La pregunta respecto a la cual entrega información este artículo refiere a la pertinencia de esta especialización o, a mantener una formación integral no sólo en los momentos de formación inicial del docente, sino también en los procesos posteriores de actualización.

¿Será la falta de especialización en ciertos grupos etáreos o áreas del conocimiento el problema? $\mathrm{O},{ }_{i}$ El problema estará constituido por la necesidad de una formación docente efectivamente integral desde una perspectiva educativa donde los contenidos comúnmente denominados instruccionales tengan el mismo valor que aquellos denominados formativos?

Hoy la investigación muestra la importancia de un cierto equilibrio entre lo instruccional y lo formativo. La investigación referida a calidad de la educación entrega evidencia que el denominado por algunos como clima escolar, por otros inteligencia emocional, y por otros valores morales de la educación tienen un impacto significativo en los aprendizajes de los niños y niñas. Sin embargo, en general los esfuerzos en los procesos de formación continua se han realizado especialmente respecto de contenidos instruccionales y aún no conocemos sus resultados.

Este artículo busca entregar alguna información para este debate, se propone enriquecer la reflexión, para entrar a analizar el tema de la posible especialización de la Educadora de Infantil desde la pregunta ¿La falta o escasa especialización en un grupo etáreo o en un área específica del conocimiento es el problema o, éste está constituido por un ámbito de la educación que ha quedado olvidado o se ha trabajado menos: lo formativo?

\section{La Educadora, el Educador: funciones en los ámbitos formativo e instruccional}

Iniciaré este capítulo con un poema, con la Oración de la Maestra, en la medida que desde la experiencia como docente de una educadora y poeta sintetiza el rol que se nos asigna:

974 Educação \& Realidade, Porto Alegre, v. 40, n. 4, p. 973-986, out./dez. 2015. 
¡Señor!Túqueenseñaste,perdonaqueyoenseñe;quelleveel nombre de maestra, que Tú llevaste por la Tierra.

Dame el amor único de mi escuela; que ni la quemadura de la belleza sea capaz de robarle mi ternura de todos los instantes.

Maestro, hazme perdurable el fervor y pasajero el desencanto. Arranca de mí este impuro deseo de justicia que aún me turba, la mezquina insinuación de protesta que sube de mí cuando me hieren. No me duela la incomprensión ni me entristezca el olvido de los que enseñe.

Dame el ser más madre que las madres, para poder amar y defender como ellas lo que no es carne de mis carnes. Dame que alcance a hacer de una de mis niñas mi verso perfecto y a dejarte en ella clavada mi más penetrante melodía, para cuando mis labios no canten más.

Muéstrame posible tu Evangelio en mi tiempo, para que no renuncie a la batalla de cada día y de cada hora por él. Pon en mi escuela democrática el resplandor que se cernía sobre tu corro de niños descalzos.

Hazme fuerte, aun en mi desvalimiento de mujer, y de mujer pobre; hazme despreciadora de todo poder que no sea puro, de toda presión que no sea la de tu voluntad ardiente sobre mi vida (Memoria..., 2014, online).

En este poema, desde la perspectiva de lo que debe ser la labor del docente quedan claras dos grandes ámbitos de funciones: instruccionales y formativas.

La primera, refiere a poner en contacto al alumno con los grandes conocimientos y avances de la humanidad, con los denominados contenidos culturales y, que en el contexto de una determinada sociedad son considerados legítimos e importantes. Esta función la ha cumplido la educación y por ende el educador desde los diversos tipos de currículum, enfatizando desde cada uno de ellos, en determinados contenidos, en unos por sobre otros. Por ejemplo, en un currículum como racionalismo académico se ha enfatizado en la trasmisión de información y desde el alumno, en su correlato: la memorización de los aportes y avances surgidos.

Por su parte, la función formativa, refiere centralmente al aporte que desde la educación y desde el docente se hace al estudiante-persona, a su proyecto como tal, a su formación valórica. También dependerá del tipo de currículum el mayor o menor énfasis que se dé a determinados aspectos del desarrollo y de la construcción de identidad de este.

Comenius, Padre de la Educación Moderna, en su obra Didáctica Magna con la cual inaugura la Pedagogía en cuanto Ciencia refiriéndose al fin de la Escuela y por ende en el contexto del propósito de la educación señala:

Llamo Escuela, que perfectamente responde a su fin, a la que es un verdadero taller de hombres; es decir, aquella en 
la que se bañan las inteligencias de los discípulos con los resplandores de la Sabiduría para poder discurrir prontamente por todo lo manifiesto y lo oculto (como dice el libro de la Sabiduría 7:17); en las que se dirijan las almas y sus afectos hacia la universal armonía de las virtudes y se saturen y embriaguen los corazones con los amores divinos de tal modo que todos los que hayan recibido la verdadera sabiduría en escuelas cristianas vivan sobre la tierra una vida celestial. En una palabra; escuelas en las que se enseñe todo a todos totalmente (Comenius, 1962, p. 37).

En este párrafo, si bien Comenius concreta el propósito de la educación en un concepto de gran fuerza taller de hombres, en el cual el joven se forma integralmente como ser humano, a la vez distingue entre lo que él denomina la inteligencia (lo instruccional) y, la sabiduría (lo formativo).

En el siguiente capítulo, luego de diagnosticar la educación de su época y señalando las características que debe tener una Escuela a fin de reformarla explicita los dos conceptos - instrucción y formación señalando:

En los cuatro párrafos que siguen vamos a demostrar que con un solo y mismo método se puede instruir y formar a una juventud [...] (Comenius, 1962, p. 37).

Como se puede observar, Comenius, ya en el siglo XVII, hace la distinción a la cual nos referimos a través del poema que inicia este texto. Si analizamos este mismo tema desde la investigación, por ejemplo Basil Bernstein distingue entre lo que él denomina el discurso regulativo, que corresponde a los valores que explícita o implícitamente trasmiten los docentes y el discurso instruccional, correspondiendo a los contenidos de las asignaturas trasmitidos por los docentes. Al respecto señala:

El discurso pedagógico siempre inserta un discurso instruccional en un discurso regulativo... Por discurso instruccional simplemente entiendo el discurso que crea competencias o habilidades especializadas. Esto puede ir desde entrenar al niño en el control de esfínteres hasta la trasmisión de la Física o de la Química. Todo esto es discurso instruccional. De manera que el discurso instruccional crea las habilidades que deben ser adquiridas y las relaciones entre ellas. El discurso regulativo hace otra cosa. Si decimos que el discurso instruccional crea orden discursivo, el discurso regulativo crea orden social. El discurso regulativo contiene las reglas que constituyen el orden, la relación y la identidad (Bernstein, 1988, p. 38).

Desde Comenius, o desde Bernstein, es necesario destacar que los dos aspectos, lo instruccional y lo formativo han estado siempre presentes en la educación y en la función que el educador o educadora 
realiza, sin embargo, a menudo, la primera se ha dado explícitamente, formulándose objetivos, evaluándose aprendizajes, desarrollándose textos de apoyo, etc. Por el contrario, la función formativa, a menudo ha permanecido en el implícito ${ }^{1}$, aportándose al desarrollo del estudiante través del estilo de relaciones que se establece con los docentes, con los compañeros, con la institución escolar, con sus reglas y reglamentos, con los espacios que cuentan niños y jóvenes para aprender, recrearse y socializar, entre otros. Pero, al quedar en el implícito, al no ser ni hacerse conciente e intencionado, la posibilidad de errar, o de dejar de asumir determinados temas que para los alumnos y alumnas son cruciales, enfrenta a profesoras y profesores a graves riesgos.

Niñas, niños y jóvenes contentos con sus docentes, que confían en ellos, que son respetados, estarán más abiertos a aprender y, a la vez, docentes contentos con sus alumnos y alumnas, que gozan con sus aprendizajes y desarrollo como personas, que disfrutan de sus éxitos, tendrán una mayor capacidad para enseñar y una mejor disposición afectiva para hacerlo.

Reconociendo quienes somos docentes, que la educación posee ambas funciones y que por el sólo hecho de ser docentes se tienen responsabilidades en los dos aspectos; para asumir lo formativo intencionadamente, nos enfrentamos a un segundo y tercer desafío.

En general, la visión de las alumnas y alumnos, qué hacer para ellos, qué enseñar, en qué y cómo orientarlos, se construye desde la teoría que se ha recibido en la Universidad y en los posteriores procesos de formación continua. Si bien, desde las observaciones, desde la información que se recaba en las fichas de matrícula los docentes poseen datos del estudiante, escasamente, sirven de sustento para las intenciones educativas-formativas y posterior desarrollo curricular.

La visión de estudiante con el cual cada docente trabaja, también se ha ido construyendo a partir de la imagen que se tiene de las familias de las/os estudiantes, al confrontar ciertas características de ellas, con la idea que el docente tiene acerca de lo que debe ser una familia. Posiblemente si el niño proviene de una familia con mamá y papá se tendrá una imagen distinta para ese alumno que si proviene de una familia en la cual es la abuela la que está a su cargo, y a la vez distinta si vive en un Hogar de Menores. La imagen también diferirá si los padres o la madre son profesionales, trabajan en una fábrica, que si lo hacen como vendedores ambulantes, o si trabaja en la prostitución, entre otras. Sin embargo, la influencia de la visión de familias y su relación con la imagen de niños o joven no sólo influye, en ello, en la imagen, sino también en las expectativas que se tengan para esos alumnos en términos instruccionales y formativos y, en cómo el docente se relaciona con ellos.

El problema se hace más complejo al generar interpretaciones respecto de los/as estudiantes a partir de las propias experiencias de los docentes; de vida, de niñez, de concepciones acerca de lo que es correc- 
to o incorrecto, bueno o malo, buena o mala conducta, buena o mala presentación persona, etc. Sin embargo, difícilmente se pone en cuestionamiento el parámetro desde el cual se juzga o se construye la visión del estudiante actual.

Podemos estar de acuerdo en la existencia de valores universales que hasta hoy perduran como la Paz, la Verdad, el Amor, etc., sin embargo existe otra serie de aspectos - que muchas veces se llaman valores - que en la época en que se desarrolló la niñez de muchos de quienes son educadoras/es se les asignaba una connotación positiva o negativa, pero que actualmente carecen de él o, han sido reemplazados por otros.

Sin embargo, al construir una determinada interpretación acerca de los niños/as y jóvenes y al no analizar críticamente el sustento desde el cual se construye dicha interpretación; sin querer, se enjuicia negativamente a los niños y jóvenes en algunos aspectos y positivamente en otros, que para los desafíos del hoy y del futuro no son los más adecuados o los más importantes. Por ejemplo, antiguamente se valoraba positivamente que los/as niños no entraran en discusión con los adultos, aunque estuvieran en desacuerdo con su postura; Si construimos desde esa valorización la interpretación que hacemos de un curso de segundo año de Secundaria, posiblemente gran parte de esos jóvenes va a ser calificado de mal educado, porque hoy se valora y promueve la actitud crítica e inquisitiva de los niños desde la Educación Infantil, se pretende fortalecer el pensamiento divergente, entre otros, incluso, desde los currículos nacionales. Otro ejemplo, hace pocos años, las diferencias claras y evidentes entre niños y niñas en todos los aspectos era un bien altamente valorado, existían claras reglas respecto del largo del pelo, de ciertos colores, de cierta ropa y adornos. Si actualmente hacemos una interpretación de lo que son los/as estudiantes de un Jardín Infantil, Escuela o Liceo específico; desde dichos parámetro una gran parte de ellos será catalogado de desordenados, descuidado, indisciplinados, pandilleros, hippies, etc. Posiblemente, para los jóvenes de hoy, dejarse el pelo largo los varones, o pelarse las niñas, o ponerse aros los muchachos y pantalones que se le caen las niñas, no es más que una moda de los jóvenes y la búsqueda de estilos propios. Sin embargo desde interpretaciones basadas en lo que se valoraba hace pocos años atrás, cuando muchos de nosotras o nosotros éramos niños o jóvenes lleva a casos tan extremos como a la suspensión de alumnos por su vestimenta, e incluso a la expulsión.

Finalmente, en la sociedad actual se han generado, o se han develado, diversidades, especificidades que sólo hace pocos años eran impensables, desconocidos o de los cuales sólo se sabía en el silencio de cuatro paredes y, respecto de los cuales se hacen interpretaciones. Trabajar en el Jardín Infantil, en la Escuela o Liceo con niños, niñas y jóvenes que viven en la calle, otros de la calle, institucionalizados, explotados sexualmente, familias monoparentales formadas por una madre tan joven como nuestros estudiantes de enseñanza Infantil, Primaria o 
Secundaria o, sólo por un padre, muchas veces lleva a interpretaciones acerca de ellos nuevamente con parámetros del pasado y, a menudo, donde la carga negativa de nuestra interpretación es más fuerte que la positiva, lógicamente, porque antes, cuando muchos de los docentes se formaron, este tipo de diversidades no existían, o eran mal valoradas, o el sentido común compartido señalaba que era malo.

Frente a una sociedad, a familiares, niños, niñas, jóvenes, comunidades diversas, desafiantes para muchos de los parámetros desde la cual la formación inicial de educadores se realizó, exige de la educación y de sus profesionales vivir procesos de permanente actualización que les facilite, adentrarse en la cotidianeidad de los alumnos /as, en sus expectativas, en sus intereses, en sus necesidades, en sus formas de pensar e interpretar, de tal forma de poder conocer y comprender a las niñas, niños y jóvenes de Brasil, Chile, Perú, Nicaragua, México hoy, especialmente a aquellos con los cuales se trabaja.

Más allá de estas diversidades propias del ser humano, un conjunto de aspectos de la sociedad actual también resultan en nuevas exigencias: el cuidado del medio ambiente, la participación como ciudadano, la cultura que se genera en el contexto del proceso de globalización, entre muchos otros.

Si a estas exigencias desde el ámbito formativo le sumamos el crecimiento exponencial del conocimiento, el acceso de los niños y niñas de hoy a este a través de las Tics, de la televisión y también a través de los medios escritos y los juguetes didácticos, el/la Educador/a debe tener una mejor y mayor preparación, continuamente enriquecida para poder responder a los desafíos que las niñas/os le plantean.

\section{La Educadora, el Educador y la Calidad Educativa: la necesidad de formación continua}

La literatura internacional muestra dos grandes aspectos relacionados con la calidad del cuidado y la Educación Infantil: a) la estructura de la clase y b) la dinámica de la clase.

\footnotetext{
Respecto de la estructura de la clase o del programa, son factores esenciales:

- un grupo apropiado y efectivo en tamaño, en ratio adulto niñola, en su composición y aspectos materiales/infraestructura, iluminación, etc.)

- soporte administrativo y de los servicios de apoyo (aseo, alimentación, etc.)

- Características del equipo que trabaja en el centro, incluye la formación inicial y la experiencia del personal docente, del director, el programa de trabajo de la dirección y del supervisor y lo apoyos que este provee.

En relación a la dinámica de la clase, son elementos centrales de calidad:
} 
- Conductas positivas de la Maestra entendidas en otros como atención al niñola, sensibilidad y responsabilidad hacia y con ellos

- Conductas positivas de los niños/as, comprendiendo su interacción con los materiales educativos, cooperación, juego, etc.

- Interacciones efectivas Maestra/o-niño, como por ejemplo interacciones verbales

- Estabilidad y continuidad (Young, 2000, p. 150).

La investigación reseñada en el texto citado (Young, 2000) señala también ocho factores que aportan a la calidad de los Programas:

- La formación inicial docente, especializada en Educación Infantil

- Capacitación en servicio respecto de educación

- La experiencia de las maestras en trabajo con niños pequeños,

- La continuidad del equipo docente en el Programa

- Compensaciones adecuadas al equipo docente y al resto del equipo que trabaja en el Programa

- Un Director del centro Infantil con experiencia y con la capacidad de apoyar y supervisar al equipo del Programa

- Coordinación con organizaciones e instituciones de la comunidad que provean otros servicios a los niños/as del programa como por ejemplo salud,

- Espacio físico adecuado y en buen estado

Así mismo, en los últimos libros de la OCDE sobre Educación Infantil (OECD, 2006), y producto de los hallazgos de una segunda y tercera ronda de evaluaciones de sus países miembros, entre los aspectos que darían cuenta de la calidad de los Programas estarían:

- Orientación a la Calidad, entendida como un marco político y legal que soporte a los Programas de educación para la Primera Infancia

- Calidad Estructural, entendida como aquellos requerimientos estructurales de infraestructura, cantidad y características del espacio físico interior y exterior, los niveles de competencia del equipo humano para el trabajo con los niños/as, el currículum, la ratio adulto-niño, las condiciones de trabajo del equipo del programa y las compensaciones adecuadas a éste

- El concepto y la práctica educativa, comprendido por los grandes fines de la Educación Infantil, el currículum, respecto de este último el que considera los cuatro pilares fundamentales planteados por el Informe Delors sería el que daría cuenta en mejor forma de este aspecto

- Interacciones y procesos cualitativos, comprendido por la calidad de la interacción pedagógica entre Maestra/o - niño/a, entre los

980 Educação \& Realidade, Porto Alegre, v. 40, n. 4, p. 973-986, out./dez. 2015. 
niños/as, entre los educadores y los grandes propósitos de la educación, entre educadores y con la dirección. El informe respecto de la relación educador niño señala:

\begin{abstract}
[...] es la más efectiva cuando la relación incluye cuidado, preocupación por el estado general de cada niño o niña, asimismo como el entregar a cada niño/a un apoyo experto para su aprendizaje. Ello integrado por un enfoque y relaciones fundadas en un concepto de pedagogía basada en la tradición de pedagogía social de los países del norte y centro de Europa [...] (OCDE, 2006, p. 127-129).
\end{abstract}

- Calidad Operacionalizada, incluye la planificación del centro, de cada grupo o nivel, oportunidad para el desarrollo continuo del equipo del centro, tiempo para observar a los niños/as, evaluación y documentación, apoyo al equipo en la forma de acompañamiento y monitoreo, inclusión de otros servicios de apoyo como salud, beneficios sociales, entre otros

- Evaluación de los resultados y del proceso educativo

- Participación de las familias, de la comunidad y el medio social y cultural, en el sentido de conocer e integrar normas, valores, y apoyar al medio en el cual el programa se encuentra

En el último informe de UNESCO sobre Atención y Educación de la primera Infancia y refiriéndose evaluativamente a los Centros Especializado de Atención y Educación de la Primera Infancia señala entre sus características positivas y sus efectos sobre mejores aprendizajes en los niños/as, entre otros, los siguientes:

Los Programas que aplican esos Centros no sólo suelen estar mejor organizados y estructurados, sino que además tienen un componente educativo más acusado.

[...] existe una sólida correlación entre programa preescolar de buena calidad (esto es, el que fomenta relaciones interactivas y cálidas con los niños y están dirigidas por docentes formados) y el mejor desarrollo intelectual y social de los niños.

[...] el desarrollo y el bienestar de los niños guarda una estrecha relación con la calidad del programa. También muestra que las interacciones entre adultos y niños son más importantes para la mejora del bienestar de estos últimos que una serie de elementos estructurales [...].

[...] los niños que a la edad de cuatro años se hallaban en contextos caracterizados por el predominio de actividades libremente escogidas consiguieron en el test de lengua efectuados a la edad de siete años puntuaciones más altas, o muchos más altas que los niños que habían estado en contextos caracterizados por actividades preescolares como lectura, escritura y matemática [...]. 
Formación Continua de los Docentes de la Educación Infantil

La frecuencia de las interacciones entre los adultos y los niños de cuatro años guarda una relación positiva con los resultados lingüísticos a la edad de siete años. [...]

[...] En los países donde predominan las actividades libremente escogidas, la frecuencia de las interacciones entre los niños de cuatro años y los adultos guarda una relación positiva con los resultados cognitivos a la edad de siete años [...] (UNESCO, 2007, p. 169-171).

En los tres libros citados, más allá del niño/a centro de preocupación de la Educación Infantil, se nos presenta como actor fundamental el docente, su formación inicial, su formación continua y desde allí el tipo de interacción que establece con los niños/as. Por ello no es casual, que nuevamente en la segunda evaluación realizada en América Latina por parte de OREALC/UNESCO se concluya:

En un mensaje alentador para todos los sistemas educativos, el SERCE ha podido constatar en su avance del estudio de factores asociados, que las escuelas pueden hacer una importante contribución al desempeño de los estudiantes. Si bien los factores de contexto socioeconómico tienen una influencia poderosa en el rendimiento, las variables asociadas a la escuela pueden contribuir significativamente a disminuir las desigualdades de aprendizaje asociadas a disparidades sociales.

En concordancia con lo observado en el PERCE, el clima escolar es la variable que mayor influencia ejerce sobre el rendimiento de los estudiantes. Por tanto, la generación de un ambiente de respeto, acogedor y positivo es esencial para promover el aprendizaje entre los estudiantes.

Las variables de recursos escolares, en conjunto, también contribuyen al rendimiento. Si bien es pequeña la contribución individual de la infraestructura, los servicios básicos de la escuela, el número de libros de la biblioteca escolar y los años de experiencia del docente, en su conjunto estas variables aportan al aprendizaje de los estudiantes. Al respecto el mensaje esencial que queda es que los recursos son necesarios para impulsar el rendimiento [...] (OREALC/UNESCO, 2007, p. 52).

En esta evaluación se confirma en la Educación Primaria lo señalado respecto de la Educación Infantil, el denominado Clima Escolar, conformado por las relaciones que se dan al interior de la sala de clases entre docentes y niños/as y, entre estos últimos es lo que mejor explica el rendimiento en matemáticas, lengua y ciencias.

Por ello no es casual, que el tema del docente de lugar a uno de los capítulos del Informe Delors y respecto de su rol se señale:

El trabajo del docente no consiste sólo en trasmitir información, ni siquiera conocimientos, sino en presentarlo en forma problemática, situándolo en un contexto y poniendo los problemas en perspectiva, de manera que el 
alumno pueda establecer el nexo entre su solución y otros interrogantes de mayor alcance. La relación pedagógica trata de lograr el pleno desarrollo de la personalidad del alumno respetando su autonomía [...] La gran fuerza de los docentes es la del ejemplo que dan al manifestar su curiosidad y su apertura de espíritu y al mostrarse dispuestos a someter a prueba de los hechos sus hipótesis e incluso a reconocer sus errores. Su cometido es ante todo el de trasmitir su afición al estudio [...] (Delors, 1998, p. 178-193).

Y luego, refiriéndose al tipo de educador mas adecuado para el trabajo con niños/as de sectores de pobreza, de medios sociales difíciles y discapacitados señala:

[...] Para tener buenos resultados, este debe poder ejercer competencias pedagógicas muy variadas y poseer cualidades humanas, no sólo de autoridad, sino también de empatía paciencia y humildad [...] (Delors, 1998, p. 178-193).

Lo que plantea de un modo abstracto el informe Delors, es lo que genera un Clima Escolar adecuado según muestra el Informe sobre la Calidad de la Educación de OREALC UNESCO, lo que la OCDE denomina El concepto y la Práctica Educativa y las denominadas Interacciones y Procesos Cualitativos y lo que Banco Mundial nomina como Dinámica de la Clase, todo ello diría relación directa con la función formativa de la educación la que a la vez influiría en la función instruccional.

Y, también los documentos citados refieren a esta última función; a lo que Young releva como interacciones efectivas entre el docente y los niños/as, al mostrar la importancia de la Especialización en Educación Infantil y la experiencia en el trabajo con niños/as. En el caso de la OCDE al señalar la importancia de aquellas competencias requeridas para la enseñanza por parte de los/as educadores, en un contexto de enfoque y relaciones desde lo que denomina una pedagogía social, la planificación de los procesos educativos y su evaluación son también actividades sustanciales para la docente. UNESCO muestra la relación entre aprendizajes de los niños/as y la existencia de un componente educativo claro en los Programas de la Primera Infancia y por ende, la necesidad de docentes formados sistemáticamente para ello Y, finalmente el Informe Delors releva la curiosidad y la disposición del docente por aprender y la apertura para poner en duda la información que maneja, abriéndose permanentemente a nuevos conocimientos.

En el marco de estos antecedentes, no basta con la formación inicial del docente, la formación continua, es una necesidad si se asume efectivamente la responsabilidad por la educación que se ofrece a los niños/as y por sus resultados en términos de aprendizaje y bienestar humano. Al respecto, en un reciente libro sobre el tema se señala:

El desarrollo profesional incluye todas las experiencias de aprendizaje natural y en las actividades conscientes y 
Formación Continua de los Docentes de la Educación Infantil

planificadas que pretenden aportar un beneficio directo o indirecto al individuo, grupo o escuela y que, a través de estos contribuyen a la calidad de la educación en el aula. Es el proceso por el cual sólo y con otros el profesorado revisa, renueva y extiende su compromiso como agente de cambio con los fines morales e la enseñanza, y por el que adquiere y desarrolla críticamente los conocimientos, destrezas e inteligencia emocional esenciales para la reflexión, la planificación y la prácticas profesionales adecuadas con los niños, los jóvenes y los compañeros en cada fase del servicio docente (Day, 2005, p. 17).

Cabe señalar nuevamente que el desarrollo profesional docente, encarnado en procesos de formación continua considera aprendizajes propios de lo que hemos denominado lo formativo y lo instruccional. No basta saber mucho de lenguaje y matemáticas, de metodologías para enseñarla y de recursos didácticos en los cuales materializar la mediación entre el contenido y aquello a aprender. Se requiere también de enriquecer al docente en cuanto persona, su inteligencia emocional, de tal modo que le facilite la relación con los niños/as, con otros docentes, con los familiares e incluso con la comunidad. Exige también observar y actuar sobre el fenómeno educativo desde una ética, en el contexto de los grandes fines de la enseñanza.

\section{Desafíos Formativos y Instruccionales para los Procesos de Formación Continua de la/el Educador/a Infantil}

Posiblemente son infinitos los temas y tensiones que debemos asignar a los procesos de formación continua, olvidaré muchos que Ud. tiene en mente, pero sólo lo señalado exige de quienes trabajan con niños/as pequeños desafíos como los siguientes:

Primero considerarse un/a profesional de la educación. Entendiendo por tal a un/a especialista que se maneja teórica y prácticamente en la educación de los niños/as pequeños y que desde allí decide sustentadamente, que a la vez hace cotidianamente ciencia (investiga, sistematiza y evalúa) para sustentar mejores decisiones y, que finalmente se maneja en una ética que tiene como eje los derechos de los niños/as y entre ellos: el derecho a aprender lo máximo y significativamente.

Segundo, interpretarse como una diseñadora de currículo, que desde fundamentos científicos surgidos desde la teoría y desde la investigación en aula diseña un currículo pertinente culturalmente, adecuado para la experiencia de los niños/as y, que considera su característica de persona, es decir un ser integral.

Tercero, comprenderse en proceso de permanente actualización y perfeccionamiento. Poseer un título de Maestro no basta, se requiere de lectura sostenida, de asistencia a cursos de reflexiones teóricas y sobre la práctica con otras Maestras/os y, a la vez de participar de otras actividades de desarrollo personal que enriquezcan el ser de quienes somos 
Maestras y Maestros: asistir a conciertos, a exposiciones pictóricas, a charlas sobre temas diversos, etc. Para ser un buen Maestro se requiere una formación integral como la que pretendemos ofrecer a los niños/as.

Cuarto, responsabilizarse además de ejercer docencia de evaluar, pero no sólo los aprendizajes de los niños/as sino también a nosotras/ os mismas/os; lo que planificamos, la metodología que utilizamos, los recursos didácticos con los cuales se concretó la actividad y, también los resultados de este conjunto en los niños/as: ¿aprendieron?, ¿cuánto?, ¿por qué? Son preguntas para el día a día de nuestro ejercicio profesional.

Quinto, actuar desde la reflexión crítica y sustentada respecto de las miles de modas que llegan a la educación. Debemos recordar que en un mundo donde todo es posible de vender también las ideas se venden. Un día somos piageteanas y al otro vigostkyanas, un día constructivistas y al otro trabajamos por competencias. Más allá de la flexibilidad para actualizarnos e incluir todo aquello que sea útil para una mejor docencia, dicha inclusión debe hacerse en el contexto de fundamentos sólidos y no en razón que alguien lo lanzó como idea o como lo último en educación. Recordemos que no necesariamente lo último es lo mejor.

Finalmente y seguramente la clave es trabajar siempre desde y para el interés superior del niño/a, para que estén integral y cualitativamente mejor, para que aprendan más y logren aprendizajes significativos, para que accedan a todas las posibilidades que el mundo actual brinda en términos de conocimientos y experiencias, para que sean mejores personas y ciudadanos, para que estén más felices y, para que a futuro contribuyan a lograr un mundo mejor: sin pobreza, sin violencia, en un ambiente armónico con la naturaleza y donde las expectativas de ser parte de una familia, de un país y de un continente se cumplan.

El desafío que tienen quienes somos Maestras/os y para quienes deciden la formación continua es grande y, posiblemente quien mejor lo refleja por haber sido Maestra y a la vez poeta es Gabriela Mistral, través del poema con el cual iniciamos esta reflexión.

Recibido en 12 de noviembre de 2014 Aprobado en 04 de mayo de 2015

\section{Nota}

1 Cabe destacar que los Objetivos Transversales, existentes en la Educación chilena y en la de otros países explicitan algunos objetivos propios de esta función.

\section{Referencias}

BERNSTEIN, Basil. Poder, Educación y Conciencia. Santiago: CIDE, 1988.

COMENIUS, Juan Amos. Didáctica Magna. Ciudad de México: Editorial Porrua, 1962.

Educação \& Realidade, Porto Alegre, v. 40, n. 4, p. 973-986, out./dez. 2015. 
DAY, Cristopher. Formar Docentes: cómo, cuándo y en qué en las cuales aprende el profesorado. Madrid: Editorial Narcea, 2005.

DELORS, Jacques. Informe a la Unesco de la Comisión Internacional sobre la Educación para el siglo XXI. In: La Educación Encierra un Tesoro. Quito: Ediciones UNESCO, 1998.

MEMORIA Chilena. Gabriela Mistral (1889-1957). Santiago: Biblioteca Nacional de Chile, 2014. Disponible en: <http://www.memoriachilena.cl/602/w3-article-3429.html>. Acceso en: 01 nov. 2014.

OECD. Starting Strong: early childhood education and care. Washington: OECD Publishing, 2006.

OREALC/UNESCO. Los Aprendizajes de los Estudiantes de América Latina y el Caribe: resumen ejecutivo del primer reporte de resultados del degundo estudio regional comparativo y explicativo. Santiago: Salesianos Impresiones, 2007.

UNESCO. Atención y Educación de la primera Infancia. Informe de Seguimiento de la EPT en el Mundo. Bélgica, 2007.

YOUNG, Mary. From Early Child Development to Human Development. Washington: World Bank, 2000.

Ofelia Reveco Vergara es doctora en Educación UAHC. Magíster en Ciencias Sociales con Mención en Sociología de la Cultura. ILADES, 1990. Educadora de Párvulos. Universidad de Chile. Actualmente Coordinadora de Investigación. U Central Chile. Se ha desempeñado como Investigadora o evaluadora por más de treinta años realizando investigaciones y evaluaciones para SCH Noruega, UNESCO/OREALC, BID, OCDE, OEA, etc.

E-mail: ofeliareveco@hotmail.com 